\title{
Mühsamer Weg zum Mainstream
}

\begin{abstract}
Vor rund vier Jahrzehnten begannen kleine Gruppen von Politik- und Umweltaktivisten auf Grasswurzel-Ebene ihre Vision von technologischer Entwicklung zu realisieren: Öko-Häuser, Windkraftanlagen und Gemüse ohne Pestizide. Heute sind manche Aspekte der Träume von damals gesellschaftliche Normalität. Eine historische Analyse der Alternativbewegung für andere Technologien liefert wertvolle Hinweise zur Durchführung von Nischenmanagement.
\end{abstract}

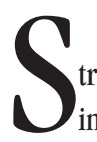
Von Adrian Smith trategisches Nischenmanagement ist - wie im Beitrag von Bernhard Truffer in diesem Heft näher dargestellt - ein neuer Ansatz der Technologiepolitik. Er zielt darauf ab, Visionen nachhaltiger sozio-technischer Praktiken als Experimente innerhalb von geschützten Nischen zu verwirklichen. Besonderes Gewicht wird dabei auf Lern- und Veränderungsprozesse von unten gelegt. Die Experimente schaffen Erkenntnisse über die Umsetzbarkeit von Nischenpraktiken, ihre Verbesserungsfähigkeit und wie sie den Mainstream verändern können. Dabei erkennt der neue Ansatz an, dass Transformationsmöglichkeiten auch außerhalb der Kontrollmöglichkeiten von Nischen-Teilnehmern liegen können. Unter Umständen müssen Nischen-Durchbrüche von übergeordneten Veränderungen im Mainstream der Gesellschaft begleitet werden.

Die Beziehung zwischen Nische und Mainstream kann ideal an einem historischen Beispiel untersucht werden: der britischen Bewegung für alternative Technologien, alternative technology movement kurz AT genannt, der siebziger Jahre.

Anhänger der AT-Bewegung haben Möglichkeiten gesucht, technologische Praktiken zu fördern, die ökologisch harmonisch und sozial verträglich sind. Die folgende Darstellung der historischen Entwicklung bezieht sich hauptsächlich auf die Entwicklung in Großbritannien, die jedoch relativ typisch für die Erfahrungen in anderen Ländern ist. Die Darstellung untersucht die hinter dem ATKonzept stehende Vision, die Strategien verschiedener AT-Anhänger und die Mechanismen, durch die die AT-Bewegung Einfluss ausgeïbt hat (1).

\section{Die Vision}

\section{alternativer Technologien}

Die AT-Bewegung war eine Reaktion auf die Umweltzerstörung in der modernen Gesellschaft. Sie war beeinflusst vom Idealismus der Studentenbe- wegung, der Gegenkultur und der neuen Linken. Anhänger der AT-Bewegung sahen Technologie als Instrument sozialer Kontrolle und gingen der Frage nach, wie alternative Technologien zur Schaffung einer sozialen und ökologisch nachhaltigen Gesellschaft beitragen könnten. AT sollte umweltfreundlich statt zerstörerisch sein, ressourceneffizient statt materialintensiv, langlebig statt zum Wegwerfen, partizipativ statt technokratisch, und statt am Profit an der Nachfrage orientiert.

Als alternative Technologien wurden zum Beispiel Windenergie, Solarheizungen, Biogas, biologische Landwirtschaft, Haus-Eigenbau, Recycling, Wärmepumpen und Wasserkraft gesehen. Einige Aktivisten vertraten allerdings die Meinung, dass die Vorgabe von alternativen Technologien der Idee von AT nicht gerecht werde. Das Ziel solle dagegen sein, alternative Prinzipien für die Wahl von Technologien zu entwickeln und sicherzustellen, dass sie den angestrebten sozialen und ökonomischen Umständen angepasst sind. Darüber hinaus gab es verschiedene Ansichten darüber, wie mit Unternehmen und Regierung umgegangen werden sollte. In der Regel konnten jedoch genügend Gemeinsamkeiten zur Bildung einer Nische gefunden werden. Die Gemeinsamkeiten schafften Kristallisationspunkte für die Bildung von Koalitionen für die För-

\section{Inserieren Sie in Ökologisches Wirtschaften!}

Sprechen Sie genau Ihre Zielgruppe an. Erscheint $5 \times$ jährlich.

Fordern Sie noch heute die Mediadaten an! Frau Dagmar Huber berät Sie gerne.

\section{Kontakt:}

Dagmar Huber

Waltherstraße 29,80337 München

Fax 089/544 18449

E-Mail huber@oekom.de derung bestimmter Bereiche, wie zum Beispiel der Windenergie. Die AT-Vision fungierte als Inspirationsquelle und beinhaltete die zentralen Leitsätze der Bewegung.

Die wichtigsten Ressourcen der AT-Anhänger waren ihre Fantasie, ihre guten Ideen und eine gewisse technische Vorbildung. Um andere Ressourcen wie Finanzmittel, Werkzeuge, Organisation, Gebäude und politischen Einfluss zu erwerben, setzten sie verschiedene Strategien ein. Welches Vorgehen gewählt wurde, hing in erster Linie von den Überzeugungen und Interessen der Aktivisten $\mathrm{ab}$, aber auch von den Möglichkeiten und Nischen, die durch Entwicklungen in der Mainstream-Gesellschaft vorgegeben wurde. Dadurch entstand eine breite Vielfalt von Aktivitäten, die entlang zweier Dimensionen unterschieden werden können: die Förderung von Techniken oder Prinzipien sowie der sozialen Aktivität auf gesellschaftlicher oder lokaler Ebene.

- Die Aktivisten, die AT als Lebensstil praktizierten, hatten den Wunsch, sich vom System zurückzuziehen und mit Hilfe von alternativen Technologien in einem weitgehend autonomen, oft ländlichen Idyll zu leben. Individuen mit Unternehmergeist gründeten Kleinbetriebe, die Produkte an den im Entstehen begriffenen umweltbewussten Konsumenten verkauften.

- Andere AT-Anhänger bauten Demonstrationszentren auf, in denen Prinzipien und Techniken alternativer Technologie erforscht, entwickelt und präsentiert werden konnten.

- Eine dritte Gruppe von AT-Anhängern setzte sich durch ihre Arbeit in Ausbildung und Forschung für die Förderung von AT ein, zum Teil organisierten sie auch politische Kampagnen.

- Eine weitere Gruppe hat schließlich breitere Unterstützung für AT mobilisiert, insbesondere durch die Gewerkschaftsbewegung und Initiativen für alternative Kommunalentwicklung.

Selbstverständlich konnten Aktivisten an einer oder mehreren dieser Tätigkeitsformen beteiligt sein, deren Dauer recht unterschiedlich war.

\section{Einfluss der alternativen Nischen}

Der Einfluss der AT-Bewegungen war am größten, wenn ihre Lösungsansätzen durch sozio-ökonomische Trends oder Schocks - wie etwa die Ölkrisen, die Verbreitung von ökologischen Werten oder Arbeitslosigkeit - plötzlich einen wichtigere Rolle auf der politische Tagesordnung einnahmen als zuvor. Zum Teil haben diese Ereignisse dazu geführt haben, dass öffentliche Gelder in die Erforschung und Entwicklung alternativer Energie- 
quellen flossen. Zum Beispiel begannen einige Kommunen Solarhäuser zu bauen und Supermärkte nahmen biologische Nahrungsmittel in ihr Angebot auf.

Die Aktivisten stellte der relative Erfolg erst mal vor ein Dilemma, denn trotz einiger Überschneidungen waren Mainstream-Interessen an alternativen Technologien letztlich von anderen Werten und Kriterien geleitet als die der Nischenaktivisten. Die den vorherrschenden Normen am nächsten stehenden Elemente von AT wurden am leichtesten in den Mainstream integriert, während diejenigen, die etablierten Interessen gegenüber die größte Herausforderung darstellten, ausgeschlossen blieben.

Die leicht integrationsfähigen Elemente der AT-Bewegung haben zur graduellen Ökologisierung des Mainstream beigetragen. Die derzeit diskutierten, auf Nischen basierenden Ansätze der Technologiepolitik eröffnen Möglichkeiten, die Debatte über radikale Nachhaltigkeit voranzubringen. Auch während sich der Mainstream in inkrementellen Schritten reformiert, sind radikale visionäre Aktivitäten auch heute noch notwendig. Eine ökologisch-orientierte Technologiepolitik sollte diesen Initiativen helfen, den Mainstream herauszufordern - nicht zuletzt durch die Bereitstellung von Ressourcen. Wie im Falle der AT-Bewegung ist es wahrscheinlich, dass einige Ideen aus geschützten Nischen im Laufe der Zeit in den Mainstream integriert werden. Ein wichtigeres Erfolgskriterium für diese Initiativen ist aber, ob es ihnen gelingt, zu einer kritischen Auseinandersetzung um Nachhaltigkeitskonzepte beizutragen.

\section{Fazit}

Die AT-Bewegung hat es damals nicht geschafft, ihre Visionen der Zukunft umzusetzen. Dennoch wurde in der Verfolgung dieser Visionen Wissen geschaffen, dass für die Verwirklichung des Nachhaltigkeitsgedankens wichtig ist und zum Teil auch heute noch Relevanz hat. In dem Moment, in dem einflussreiche Gruppen gesellschaftliche Probleme anerkennen, können innovative Ideen umgesetzt werden - auch wenn ihre radikaleren Elemente dabei oft ausgeblendet werden. Es wäre allerdings naiv anzunehmen, dass dies ein unvermeidbarer und unumkehrbarer Prozess ist. Nischen alleine werden die, die an der Spitze existierender Machtstrukturen stehen, nicht dazu bringen, sich einem radikal-ökologischen Nachhaltigkeitsverständnis anzuschließen. Die Prozesse, durch die andere Gruppen beginnen, sich für solche Ideen zu interessieren, sie zu nutzen und weiterzuverbreiten, sind oft außerhalb der Kontrolle von Nischen-Pionieren. Die Geschichte der AT-Bewegung macht deutlich, wie innovative Ideen aus der Zivilgesellschaft hervorgehen. Ihr Einfluss kann von gesellschaftlichen Trends und Entwicklungen sowohl gefördert als auch eingeengt werden.

Die Geschichte macht auch deutlich, dass das Bild der Nischen mit Bedacht verwendet werden sollte. Es sollte keine dichotomische Beziehung suggerieren, in dem die Nische als grüner Tropfen gesehen wird, der gleichmäßig in den Mainstream diffundiert und ihn hellgrün verfärbt. Die Beziehung zwischen Umweltpionieren und anderen gesellschaftlichen Gruppen sind in der Praxis mehrdimensional und Ökologisierungsprozesse verlaufen ungleichmäßig. Manche Nischenakteure treffen beispielsweise die Entscheidung, mit bestimmten Interessengruppen nicht zu kooperieren. Manche etablierten Firmen sind führend an der Umsetzung von Nachhaltigkeitsideen beteiligt, während andere energischen Widerstand leisten. Außerdem ist die Einflussnahme keineswegs nur einseitig. Nischenaktivitäten sind immer auch abhängig von den Möglichkeiten, die Gesellschaft und Wirtschaft ihnen bieten. Die Typologie der ATStrategien veranschaulicht diese vielfältigen Arten der Einflussnahme.

Obwohl die AT-Bewegung aus dem Radikalismus der sechziger Jahre hervorgegangen ist, solle man nicht vergessen, dass dieses Jahrzehnt eine noch viel einflussreichere Revolution vorangetrieben hat: den Anstieg der Konsumkultur. Millionen Verbraucher in reichen Ländern erfreuen sich eines nie da gewesenen materiellen Konsums und weitere Millionen in armen Ländern hoffen darauf, in Zukunft daran teilhaben zu können. Die AT-Bewegung war ein früher Versuch, die Herausforderung anzunehmen, die die Verbraucherrevolution mit sich gebracht hat.

\section{Anmerkung}

(1) Herzlichen Dank an Julia Hertin für die Übersetzung dieses Artikels aus dem Englischen.

\section{Der Autor}

Dr. Adrian Smith ist Wissenschaftler bei SPRU - Science \& Technology Policy Research.

Kontakt: SPRU, Freeman Centre, University of Sussex, Brighton BNI 9QE, UK. Tel. 0044-1273-

877065, E-Mail: A.G.Smith@sussex.ac.uk

\section{Unternehmen und Umwelt}
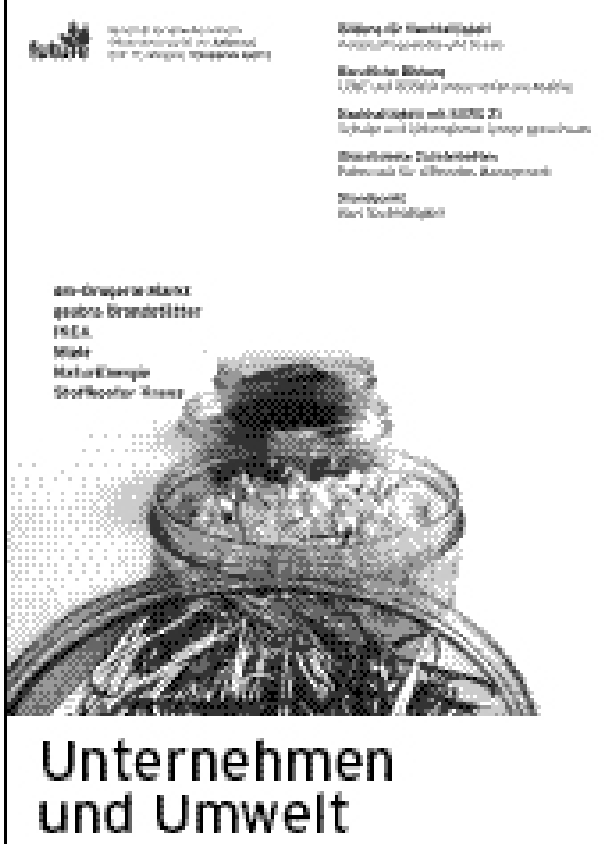

... ist die Zeitschrift für umweltorientierte Unternehmerspoltik von future e.V.

... bietet Fachinfonmationen zum Urrweltschutz und Best-Practice-Beipiek ats Unternetomen.

... bietet viermal im Jahr Untemehmersbeispick, Literatur- und Verarataltungshirweise.

Mit einem Sctwerpunktthems in jedem Heft. Zum Beisiel:

1/04: Bid deng fir Nachthaltige Entwicklung 4/03: Kampetenz nacthasitig entwickeln

3/03: Nactivaltigkeit und Regrion

2/03: Kaspital fï Nachhalitgheit

1/03: Energieeffizienz

3-4/OO2: Benctwarting for Sustainability

2/02: Obomarketing

1/02: Nachinaltigkeit und Gewerbebus

4/01: Nachinaltigkeit in Handiwerk

3/01: Klimaschutz

Bestellen Sie ihr kostenloses

Probe-Abo im Internet unter

ww w. future-ev.de

future e.V. Büro Bochum

Am Varenholt 123

44797 Bochum

Fon: Q234/9799513

Fax: 0234/9799514

bodhumfluture-erde

www. futureev.de 
(c) 20I0 Authors; licensee IÖW and oekom verlag. This is an article distributed under the terms of the Creative Commons Attribution Non-Commercial No Derivates License (http://creativecommons.org/licenses/by-nc-nd/3.o/), which permits unrestricted use, distribution, and reproduction in any medium, provided the original work is properly cited. 